\title{
Optimization the shape of the Francis hydraulic turbine
}

\author{
Valerij Kurir $^{1}$ \\ ${ }^{1}$ Department of Electrical Equipment Kazan National Research Technical University named after A.N. Tupolev, Kazan, Russia
}

\begin{abstract}
The article presents one of the possible methods for optimizing the blade shape of a Francis radial-axial hydraulic turbine. The method for optimizing the shape of the turbine blade is based on the criterion of the maximum mechanical moment developed by the turbine. The blade shape optimization operation is conventionally divided into two stages. At the first stage of optimization of the blade shape, the analytical expression of the moment developed by the turbine is presented in a Taylor series by variable parameters - the coordinates of the vertices of the characteristic polyhedron of the median surface of the turbine blade. Adding the boundary conditions in the formulation of the optimization problem in the form of equalities - the contact of the median surface of the turbine blade with the turbine hub and rim, as well as conditions in the form of inequalities - the concavity of the greater part the median surface allows to reduce the problem of optimizing the blade shape to a standard linear programming problem. It is proposed to carry out 50-60 similar operations with small steps in the variables - the coordinates of the vertices of the characteristic polyhedron. Thus, it is necessary to move into the zone of optimal values of the coordinates of the vertices of the characteristic polyhedron of the median surface of the blade. At the second stage, it is proposed to continue the search for the optimal values of the coordinates of the vertices of the characteristic polyhedron of the median surface of the blade, applying for this purpose one of the most effective algorithms of genetic optimization.
\end{abstract}

\section{Introduction}

The design methodology for hydraulic turbines with high technical and economic performance was developed and constantly improved by Russian and foreign developers and designers for several decades. The optimal shape of the blades of hydraulic turbines (HT) with high cavitation properties were determined at that time.

The use of numerical methods, as well as cluster technologies that allow parallelizing solutions that require a fairly large counting time, allowed us to consider a number of optimization problems that were not previously available.

In the research practice of universities, research institutes, it has begun to practice multi-criterial optimization of the structures under study, as well as the elements of these structures [1 - 14].

The following are used as criteria for optimizing HT:

1. Maximum efficiency of power on the HT shaft.

2. The minimum of the ratio of the surface of HT, subject to cavitation, to the total surface of the HT.

In fig. 1 shows the elements of the hydraulic turbine - a supply pipe, a spiral case, a hydraulic turbine and a draft tube. In fig. 2 shows a general view of the Francis turbine blade.

The dominant method in the optimization of turbomachines is genetic methods.

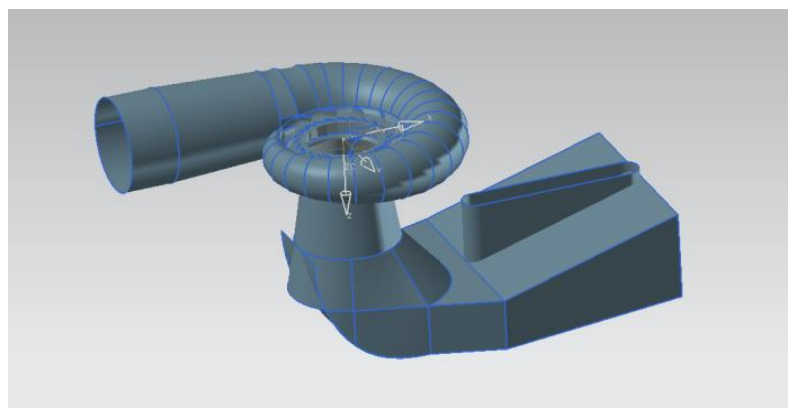

Fig. 1. Hydraulic turbine with a spiral case and draft tube.

\section{Problem statement}

Let's consider an algorithm for constructing a HT blade using computer graphics.

The construction of the blade surface begins with the construction of its median surface. We define the median surface of the blade in the form of a double cubic Bezier polynomial in variables $u, v$ in the form:

$$
\begin{aligned}
& \mathbf{r}(u, v)=\sum_{i=0}^{3} \sum_{j=0}^{3} \mathbf{r}_{i j} \frac{3 ! \cdot 3 !}{(3-i) ! \cdot i ! \cdot(3-j) \cdot j !} \times \\
& u^{i}(1-u)^{3-i} v^{j}(1-v)^{3-j},
\end{aligned}
$$


where $\mathbf{r}_{i j}$ - are the vertices of the characteristic polyhedron, $u, v$ - parameters varying from 0 to 1 .

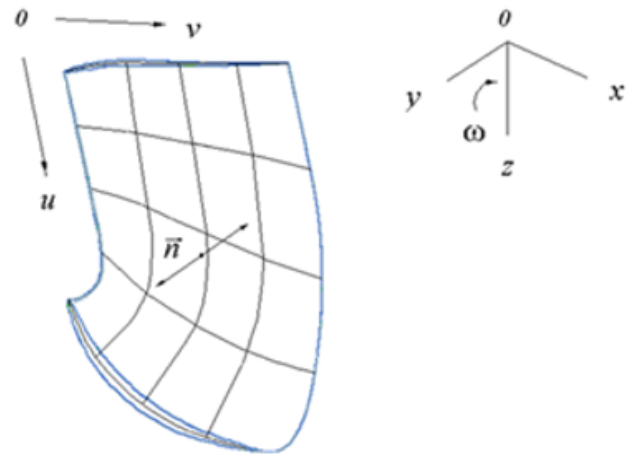

Fig. 2. General view of the blade with the applied mesh.

The inner and outer surfaces of the blade can be constructed by means of dependencies:

$$
\begin{aligned}
& \mathbf{r}(u, v)_{\text {inner }}=\mathbf{r}(u, v)+d(u, v) \cdot \underset{\mathbf{n},}{\mathbf{r},} \\
& \mathbf{r}(u, v)_{\text {outer }}=\mathbf{r}(u, v)-d(u, v) \cdot \mathbf{r},
\end{aligned}
$$

where $d(u, v)$ - is the half thickness of the blade at a given point, $\mathbf{n}$-internal normal to the median surface.

As the optimal profile of the turbine blade, we consider the blade profile that provides the maximum efficiency of the turbine during its operation.

To determine the optimal blade profile, you need to find the coordinates of the vertices of the characteristic polyhedron of the median surface of the blade $x_{i j}, y_{i j}, z_{i j}$, which provides the maximum value of the mechanical moment acting from liquid on the blade:

$$
\begin{aligned}
\max M_{Z}= & \max \left(-\int_{0}^{1} \int_{0}^{1} \mathbf{r}(u, v)_{\text {inner }} \times \mathbf{P}_{n} \cdot|\mathbf{G}|^{1 / 2} d u d v-\right. \\
& \left.-\int_{0}^{1} \int_{0}^{1} \mathbf{r}(u, v)_{\text {outer }} \times \mathbf{P}_{n} \cdot|\mathbf{G}|^{1 / 2} d u d v\right)_{Z} .
\end{aligned}
$$

Here $\quad \mathbf{r}(u, v)=\mathbf{i} \cdot x(u, v)+\mathbf{j} \cdot y(u, v)+\mathbf{k} \cdot z(u, v) ;$ $-\mathbf{P}_{n}(u, v)$ - the static pressure of water masses at the surface of the hydraulic turbine; $|\mathbf{G}|^{1 / 2} d u d v$ - the area of elementary sections of the turbine surface, where

$$
|\mathbf{G}|=\left|\frac{\partial \mathbf{r}}{\partial u}\right|^{2} \cdot\left|\frac{\partial \mathbf{r}}{\partial v}\right|^{2}-\left(\frac{\partial \mathbf{r}}{\partial u} \cdot \frac{\partial \mathbf{r}}{\partial v}\right)^{2}
$$

Expression of the normal to the surface (Fig.3) has the form where the derivative is calculated at the point $u=u_{0}, v=v_{0}$

$$
\mathbf{n}= \pm\left(\frac{\partial \mathbf{r}}{\partial u} \times \frac{\partial \mathbf{r}}{\partial v}\right) /\left|\frac{\partial \mathbf{r}}{\partial u} \times \frac{\partial \mathbf{r}}{\partial v}\right|
$$

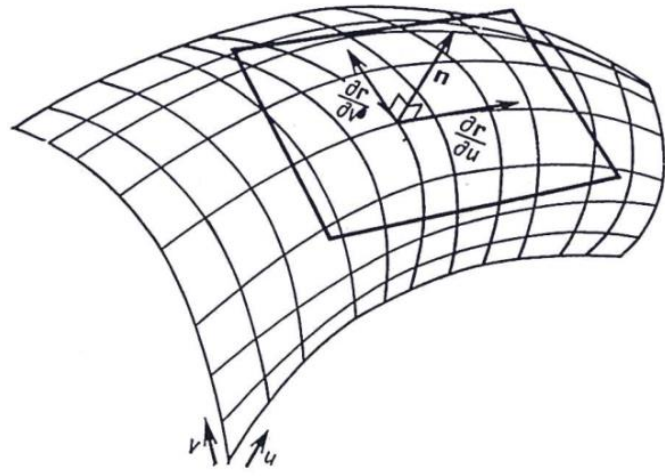

Fig. 3. Normal to surface.

Then, taking into account expressions $(1-5)$, we obtain an integrand of the form

$$
\begin{aligned}
& \underset{\mathbf{n}}{\mathrm{r}} \times\left.\stackrel{\mathrm{r}}{\mathbf{r}}\right|_{Z} \cdot|\mathbf{G}| \cdot P(u, v)=\left.\left[\frac{\partial \mathrm{r}}{\partial u} \times \frac{\partial \mathrm{r}}{\partial v} \times \underset{\mathbf{r}}{\mathbf{r}}\right]\right|_{Z} \cdot P(u, v)= \\
& \left.\frac{\partial \overrightarrow{\mathbf{r}}}{\partial v}\right|_{Z} \cdot\left(\frac{\partial \mathbf{r}}{\partial u} \cdot \mathbf{r}\right) \cdot P(u, v)-\left.\mathbf{r}\right|_{Z} \cdot\left(\frac{\partial \mathbf{r}}{\partial u} \cdot \frac{\partial \mathbf{r}}{\partial v}\right) \cdot P(u, v)= \\
& =\frac{\partial z}{\partial v} \cdot\left(\frac{\partial \mathbf{r}}{\partial u} \cdot \mathbf{r}\right) \cdot P(u, v)-z \cdot\left(\frac{\partial \mathbf{r}}{\partial u} \cdot \frac{\partial \mathbf{r}}{\partial v}\right) \cdot P(u, v)
\end{aligned}
$$

The array of static pressure values included in the expression (1) is borrowed from the solution of the hydrodynamic problem of flow around the turbine blade.

The integration size free step for variable $v$ is 0.005 , for variable $u-0.0025$.

There are two ways to optimize the blade geometry.

\section{Basics of optimizing the shape of a turbine blade}

The optimization of the blade geometry is supposed to be perfomed using a program that implements the linear programming method. This program, - fmincon, is included in the MATLAB package [15].

We believe that the moment created by the turbine blade is approximately described by a linear model of the form (the first terms of the expansion of the function (3) in the Taylor series):

$$
\begin{aligned}
& M_{z}=M_{z 0}+\sum_{i=1}^{4} \sum_{j=1}^{4}\left(M_{i j 1} \Delta x_{i j}+M_{i j 2} \Delta y_{i j}+M_{i j 3} \Delta z_{i j}\right)= \\
& =M_{z 0}^{\prime}+\sum_{i=1}^{4} \sum_{j=1}^{4}\left(M_{i j 1} x_{i j}+M_{i j 2} y_{i j}+M_{i j 3} z_{i j}\right),
\end{aligned}
$$

where $M_{z 0}-$ is the mechanical moment developed by the turbine blade (according to the hydrodynamic calculation data);

$$
M_{i j 1}=\partial M_{z} / \partial x_{i j}, M_{i j 2}=\partial M_{z} / \partial y_{i j}, M_{i j 3}=\partial M_{z} / \partial z_{i j}-
$$

partial derivatives of the expression of the moment (3) taken according to the coordinates of the vertices of the 
characteristic polyhedron of the median surface of the blade;

$$
\Delta x_{i j}=x_{i j}-\bar{x}_{i j}, \Delta y_{i j}=y_{i j}-\bar{y}_{i j}, \Delta z_{i j}=z_{i j}-\bar{z}_{i j},
$$

where $\bar{x}_{i j}, \bar{y}_{i j}, \bar{z}_{i j}$ - the coordinates of the vertices of the characteristic polyhedron obtained in the previous iteration, $x_{i j}, y_{i j}, z_{i j}$ - the coordinates of the vertices are polyhedron, determined during the period of a new iteration.

The admissible variations of the coordinates of the vertices of the characteristic polyhedron are given by the relation:

$$
\begin{aligned}
& -0,01 \mathrm{\textrm {M }} \leq \Delta x_{i j} \leq 0,01 \mathrm{\textrm {M }}, \\
& -0,01 \mathrm{\textrm {M }} \leq \Delta y_{i j} \leq 0,01 \mathrm{\textrm {M }}, \\
& -0,01 \mathrm{\textrm {M }} \leq \Delta z_{i j} \leq 0,01 \mathrm{M} .
\end{aligned}
$$

We add equations to the model connecting the coordinates of four points of the upper boundary of the blade lying on the hub and the coordinates of four points of the lower boundary of the blade lying on the rim of the turbine (Fig. 4, 5, 6):

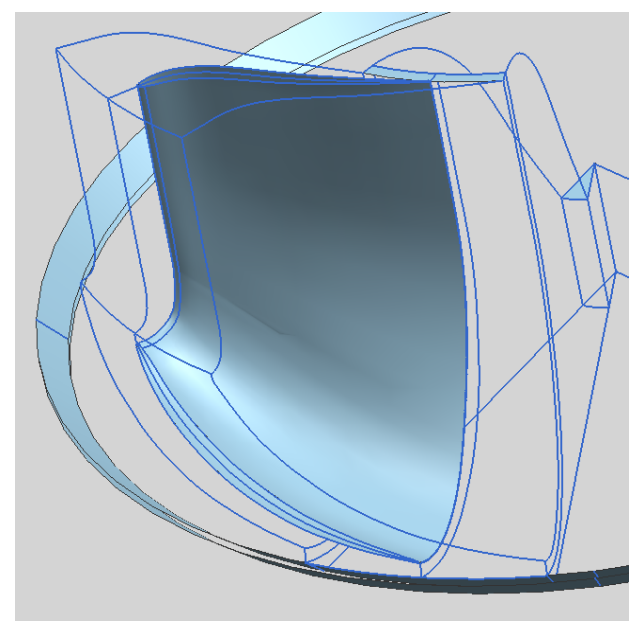

Fig. 4. The upper side surface - hub,. lower side surface - rim

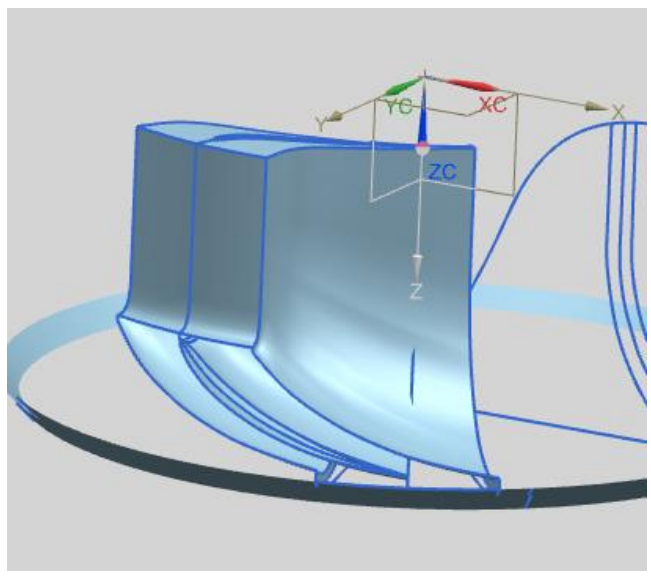

Fig. 5. The upper side surface - hub, lower side surface - rim
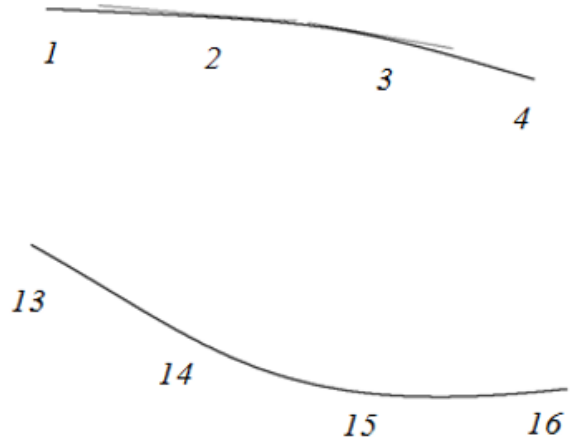

Fig. 6. The upper curve is border with hub, the lower curve is the border with the rim

$$
\begin{aligned}
& \left.z_{1}\right|_{v=0, u=0}=0,\left.\quad \sqrt{x_{1}^{2}+y_{1}^{2}}\right|_{v=0, u=0}=R_{\max } ; \\
& z=k_{1}\left(R_{\max }-R_{2}\right)=\left.k_{1}\left(R_{\max }-\sqrt{x^{2}+y^{2}}\right)\right|_{v=1 / 3, u=0}, \\
& k_{1}=\text { const; } \\
& z=k_{2}\left(R_{\max }-R_{3}\right)=\left.k_{2}\left(R_{\max }-\sqrt{x^{2}+y^{2}}\right)\right|_{v=2 / 3, u=0}, \\
& k_{2}=\text { const; } \\
& \left.z_{4}\right|_{v=1, u=0}=\text { const } 1,\left.\sqrt{x_{4}^{2}+y_{4}^{2}}\right|_{v=1, u=0}=R_{\min } ; \\
& \left.z_{13}\right|_{v=0, u=1}=\text { const } 2 ;\left.\quad x_{13}\right|_{v=0, u=1}=\text { const } 3 ; \\
& \left.\mathrm{y}_{13}\right|_{v=0, u=1}=\text { const } 4 ; \\
& \sqrt{x^{2}+y^{2}}-R_{0 \min }=\left.\left(z-Z_{0}\right) \operatorname{tg} \alpha\right|_{v=1 / 3, u=1} ; \\
& \sqrt{x^{2}+y^{2}}-R_{0 \min }=\left.\left(z-Z_{0}\right) \operatorname{tg} \alpha\right|_{v=2 / 3, u=1} ; \\
& \left.z_{16}\right|_{v=1, u=1}=\text { const5; }\left.\quad x_{16}\right|_{v=1, u=1}=\text { const6; } \\
& \left.y_{16}\right|_{v=1, u=1}=\text { const7. }
\end{aligned}
$$

Here $R_{\max }-$ the distance from axis $0 \mathrm{Z}$ to the left upper edge of the blade (Fig. 4), $\quad R_{\min }-$ the distance from axis $0 \mathrm{Z}$ to the right upper edge of the blade; $R_{0 \text { min }}$ - the distance from the axis $0 Z$ to the top border of the rim (Fig. 4, 5), $Z_{0}$-its vertical coordinate in the coordinate system $0 \mathrm{XYZ}, R_{0 \max }-$ the distance from the axis $0 \mathrm{Z}$ to the lower border of the rim, $\operatorname{tg} \alpha$ is the angle of inclination of the rim surface with respect to the axis 0Z. The diameter of the lower border of the rim is greater than the diameter of the upper border of the hub.

For a curve contacting with the hub (see Fig. 2, 4, 5) at points 1,4 , the concavity condition of the curve must be satisfied:

$$
\begin{array}{ll}
y_{v}^{\prime \prime} x_{v}^{\prime}-y_{v}^{\prime} x_{v}^{\prime \prime}>0 \quad\left(y_{x}^{\prime \prime}>0\right) \text { at point } 1, \\
y_{v}^{\prime \prime} x_{v}^{\prime}-y_{v}^{\prime} x_{v}^{\prime \prime} \geq 0 \quad\left(y_{x}^{\prime \prime} \geq 0\right) \text { at point } 4,
\end{array}
$$

where values $x_{v}^{\prime}$ and $y_{v}^{\prime}$ are taken from the previous iteration.

For a curve lying on the rim, the curve convexity condition must be satisfied: 


$$
\begin{aligned}
& z_{v}^{\prime \prime} x_{v}^{\prime}-z_{v}^{\prime} x_{v}^{\prime \prime}<0 \quad\left(z_{x}^{\prime \prime}<0\right) \text { at point } 13, \\
& z_{v}^{\prime \prime} x_{v}^{\prime}-z_{v}^{\prime} x_{v}^{\prime \prime} \leq 0 \quad\left(z_{x}^{\prime} \leq 0\right) \text { at point } 16,
\end{aligned}
$$

where values $x_{v}^{\prime}$ and $z_{v}^{\prime}$ are taken from the previous iteration.

The projections of the leading edge and trailing edge on X0Y plane are considered straight lines.

For the leading edge, the curve convexity condition must be satisfied:

$$
\begin{aligned}
& z_{v}^{\prime}>0, \quad z_{v}^{\prime \prime} r_{v}^{\prime}-z_{v}^{\prime} r_{v}^{\prime \prime}<0\left(z_{r}^{\prime \prime}<0\right) \text { at point } 1, \\
& z_{v}^{\prime}=0, \quad z_{v}^{\prime \prime} r_{v}^{\prime}-z_{v}^{\prime} r_{v}^{\prime \prime}<0\left(z_{r}^{\prime \prime}<0\right) \text { at point } 13,
\end{aligned}
$$

where values $r_{v}^{\prime}$ and $r_{v}^{\prime \prime}$ are taken from the previous iteration, $r=\sqrt{x^{2}+y^{2}}$.

For the trailing edge, the curve convexity condition must be satisfied:

$$
\begin{aligned}
& z_{v}^{\prime}>0, \quad z_{v}^{\prime \prime} r_{v}^{\prime}-z_{v}^{\prime} r_{v}^{\prime \prime}<0\left(z_{r}^{\prime \prime}<0\right) \text { at point } 4, \\
& z_{v}^{\prime}=0, \quad z_{v}^{\prime \prime} r_{v}^{\prime}-z_{v}^{\prime} r_{v}^{\prime \prime}<0\left(z_{r}^{\prime \prime}<0\right) \text { at point } 16,
\end{aligned}
$$

where values $r_{v}^{\prime}$ and $r_{v}^{\prime \prime}$ are taken from the previous iteration.

The system of equations (7) can be easily linearized using the property

$$
\Delta x_{i j}<<x_{i j}, \Delta y_{i j}<<y_{i j}, \Delta z_{i j}<<z_{i j} .
$$

We consider the coordinates of both lower corner vertices of the median surfaces to be known, given in advance. The upper left corner and right corner vertices have degrees of freedom - some rotation about the $0 \mathrm{Z}$ axis (up to 15 degrees in the clockwise direction).

We also assume that the median surface of the blade (Fig. 7) is a concave surface. That is, the product of the main curvatures of surface and the sum of the main curvatures of the surface (12) are strictly positive [16].

We check this property at points: $(u=0.25, v=0.25)$,

$(u=0.25, v=0.75),(u=0.5, v=0.5),(u=0.75, v=0.25)$, $(u=0.75, \mathrm{v}=0.75)$.

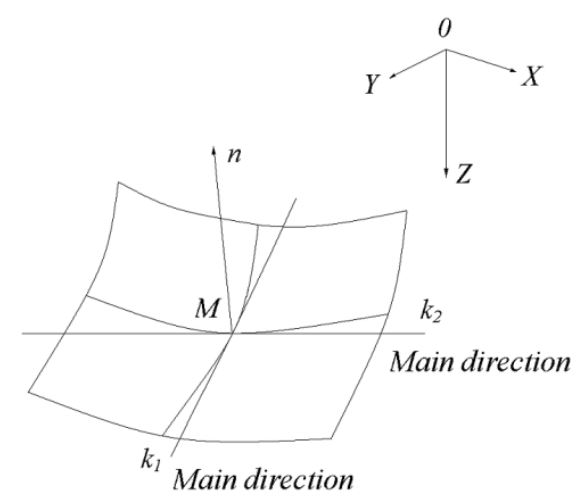

Fig. 7. Inner normal to the median surface and main directions

$$
L N-M^{2}>0, \quad E N+L G-2 M F>0,
$$

where

$$
E=\mathbf{r}_{u}^{2}=x_{u}^{2}+y_{u}^{2}+z_{u}^{2},
$$$$
F=\mathbf{r}_{u} \mathbf{r}_{v}=x_{u} x_{v}+y_{u} y_{v}+z_{u} z_{v},
$$$$
G=\mathbf{r}_{v}^{2}=x_{v}^{2}+y_{v}^{2}+z_{v}^{2} \text {. }
$$

$L=\frac{\left|\begin{array}{lll}x_{u и} & y_{\text {ии }} & z_{u и} \\ x_{u} & y_{u} & z_{u} \\ x_{v} & y_{v} & z_{v}\end{array}\right|}{\sqrt{E G-F^{2}}}, M=\frac{\left|\begin{array}{lll}x_{u v} & y_{u v} & z_{u v} \\ x_{u} & y_{u} & z_{u} \\ x_{v} & y_{v} & z_{v}\end{array}\right|}{\sqrt{E G-F^{2}}}, N=\frac{\left|\begin{array}{lll}x_{v v} & y_{v v} & z_{v v} \\ x_{u} & y_{u} & z_{u} \\ x_{v} & y_{v} & z_{v}\end{array}\right|}{\sqrt{E G-F^{2}}}$.

Here, the values $E, F, G$ and first derivatives in $L, M, N$ are calculated according to the previous iteration. The first expression (12) splits into two identical expressions (12a) and (12b), in which one of the values $M$ is calculated by the previous iteration. In expression (12a) the value $N$, in expression (12b) the value $L$ is calculated according to the previous iteration. Expresion (12a) and (12b) are used together in iterations.

Solving the problem of finding the maximum (3) when perfoming the linearization conditions (1-12), we determinate the required $\Delta x_{i j}, \Delta y_{i j}, \Delta z_{i j}$.

The resulting values for one of there increments for the hub and rim are refined from the geometry of hub and rim.

The case of local violations of conditions (12), we carry out the operation of smoothing curves, and then surfaces.

As result, we obtain the refined values of the coordinates of the vertices of the characteristic polyhedron, which make it possible to describe the new median surface of the blade:

$$
\mathbf{r}_{i j n e w}=\mathbf{r}_{i j}+\Delta \mathbf{r}_{i j} .
$$

The second way of describing the median surface of the blade is the Ferguson bicubic polynomials used to construct the Koons surface [17] .

In this case, the calculations involve the coordinates of the corner points of the middle surface, the tangent vectors at the ends of the corresponding boundary curves and the torsion vector at the corners of the surface:

$$
\begin{aligned}
& Q(u, v)=\left[\begin{array}{llll}
F_{1}(u) & F_{2}(u) & F_{3}(u) & F_{4}(u)
\end{array}\right] \times \\
& \times\left[\begin{array}{llll}
\mathbf{P}(0,0) & \mathbf{P}(0,1) & \mathbf{P}_{v}(0,0) & \mathbf{P}_{v}(0,1) \\
\mathbf{P}(1,0) & \mathbf{P}(1,1) & \mathbf{P}_{v}(1,0) & \mathbf{P}_{v}(1,1) \\
\mathbf{P}_{u}(0,0) & \mathbf{P}_{u}(0,1) & \mathbf{P}_{u v}(0,0) & \mathbf{P}_{u v}(0,1) \\
\mathbf{P}_{u}(1,0) & \mathbf{P}_{u}(1,1) & \mathbf{P}_{u v}(1,0) & \mathbf{P}_{u v}(1,1)
\end{array}\right]\left[\begin{array}{c}
F_{1}(v) \\
F_{2}(v) \\
F_{3}(v) \\
F_{4}(v)
\end{array}\right],
\end{aligned}
$$

where $\quad F_{1}(t)=2 t^{3}-3 t^{2}+1, F_{2}(t)=-2 t^{3}+3 t^{2}$, $F_{3}(t)=t^{3}-2 t^{2}+e, \quad F_{4}(t)=t^{3}-t^{2}$, where $t$ is either $u$, or $v$, respectively. 


$$
\begin{aligned}
& {\left[\begin{array}{lllr}
\mathbf{P}(0,0) & \mathbf{P}(0,1) & \mathbf{P}_{v}(0,0) & \mathbf{P}_{v}(0,1) \\
\mathbf{P}(1,0) & \mathbf{P}(1,1) & \mathbf{P}_{v}(1,0) & \mathbf{P}_{v}(1,1) \\
\mathbf{P}_{u}(0,0) & \mathbf{P}_{u}(0,1) & \mathbf{P}_{u v}(0,0) & \mathbf{P}_{u v}(0,1) \\
\mathbf{P}_{u}(1,0) & \mathbf{P}_{u}(1,1) & \mathbf{P}_{u v}(1,0) & \mathbf{P}_{u v}(1,1)
\end{array}\right]=} \\
& =\left[\begin{array}{lc}
\mathbf{r}_{0,0} & \mathbf{r}_{0,3} \\
\mathbf{r}_{3,0} & \mathbf{r}_{3,3} \\
3\left(\mathbf{r}_{1,0}-\mathbf{r}_{0,0}\right) & 3\left(\mathbf{r}_{1,3}-\mathbf{r}_{0,3}\right) \\
3\left(\mathbf{r}_{3,0}-\mathbf{r}_{2,0}\right) & 3\left(\mathbf{r}_{3,3}-\mathbf{r}_{2,3}\right)
\end{array}\right. \\
& 3\left(\mathbf{r}_{0,1}-\mathbf{r}_{0,0}\right) \quad 3\left(\mathbf{r}_{0,3}-\mathbf{r}_{0,2}\right) \\
& 3\left(\mathbf{r}_{3,1}-\mathbf{r}_{3,0}\right) \quad 3\left(\mathbf{r}_{3,3}-\mathbf{r}_{3,2}\right) \\
& 9\left(\mathbf{r}_{0,0}-\mathbf{r}_{1,0}-\mathbf{r}_{0,1}+\mathbf{r}_{1,1}\right) \quad 9\left(\mathbf{r}_{0,2}-\mathbf{r}_{1,2}-\mathbf{r}_{0,3}+\mathbf{r}_{1,3}\right) \\
& \left.9\left(\mathbf{r}_{2,0}-\mathbf{r}_{3,0}-\mathbf{r}_{2,1}+\mathbf{r}_{3,1}\right) \quad 9\left(\mathbf{r}_{2,2}-\mathbf{r}_{3,2}-\mathbf{r}_{2,3}+\mathbf{r}_{3,3}\right)\right]
\end{aligned}
$$

\section{Algorithm for finding the optimal shape of blades}

The algorithm for finding the optimal blade shape is as follows. The basic software environment in which it is assumed to carry out the process of optimization the blade shape, author adopted the MATLAB package. This package organizes an iterative cycle of launching the following packages.

1. Launching NUMECA IGG package. Preliminary creation in the package of the geometry of the Draft Tube (one-time) and application of a 3D mesh in Draft Tube (one-time) before the start of the iterative process of the turbine blade profile optimization.

2. Launching the NX package. Automatic creation of a fixed vane - Stay Vane, two adjustable vanes - Guide Vanes, a new shape of turbine blade. The graphic creation of the turbine blade, which is changed during the solution process, is implemented in the form of a script created in the Visual Basic language. Converting the resulting geometry to Parasolid format and exporting them.

A similar operation, can be perfomed in NUMECA AUTOBLADE package.

3. Launching the NUMECA FINE/TURBO package. Import specified files. Automatic 3D mesh in the NUMECA AUTUGRID mesh generator:
A. In the cell adjacent to the Stay Vane.
B. In the 2 cells adjacent to the Guide Vanes.
C. In the cell of the turbine blade.

Automatic application of the 3D mesh in these cells is implemented based on a script created in the PYTHON language.

4. Launching the ANSYS CFX package. Import the specified zones of the turbine into ANSYS CFX PRE. Statement of boundary conditions. Launching of the solution in ANSYS CFX SOLUTION. The sequence of these operations implemented in the Java Script language has been repeatedly presented in a number of articles. Switch to ANSYS CFX POST. Output of an array of calculated values of static pressure at given points of the blade surface to a file with the extension dat.

A similar operation can be perfomed in the EURANUS (NUMECA) package.

5. We read data from a file with the extension dat. Numerical integration of the surface integrals given in $(3,6)$ is carried in the MATLAB package.

6. Solving the optimization problem $(1-12)$ is carried in MATLAB package using fmincon program. We get the corrected values $\mathbf{r}_{i j n e w}=\mathbf{r}_{i j}+\Delta \mathbf{r}_{i j}$, allowing to create a new turbine blade. We calculate the value mechanical moment developed by the turbine, the efficiency of the turbine. Go to the next iteration (go to step 2).

This algorithm is considered by the author as a "starting" one, designed for $50 \div 60$ iterations. Further optimization of the blade shape should take place using genetic methods.

A similar operation for optimizing the blade shape of the Francis turbine can be perfomed in the NUMECA FINE/ DESIN 3D package.

The author suggests using the NSGA-III genetic algorithm software for these purposes. The number iterations required to complete the task is no more than 200.

\section{Method with using the sliding tolerance technique}

This solution method is based on the application of the Nelder-Mead flexible polyhedron method in the metod of conditional optimization - sliding tolerance [15].

The conditions in the form of equalities (inequalities) (7-12) for carrying out the problem optimizing the blade shape of Francis turbines remain the same.

the conditional optimization of the shape of the HT blade remain the same.

Since in [15] there is flexiplex program for perfoming conditional optimization using the sliding tolerance method with the use of minimizing the target function is given, we use the standard transition for the target function:

$$
f_{\text {max }} \rightarrow(-f)_{\text {min }}
$$

\section{Conclusion}

A new algorithm is presented that allows optimize the shape of the Francis radial-axial turbine blade in two stages, as well as a diagram of the software optimization of the turbine blade profile which is different from the existing one. To optimize the turbine profile at the first stage, the objective function, a system of equations and conditions, as well as a method for carrying out the operation of optimizing the turbine blade profile are presented - the classical method of linear programming. At the second stage of optimization of the turbine blade profile, it is proposed to use genetic optimization 
algorithms. The system for checking the geometry of the median surface of the blade $(8-12)$ for concavity, in a transformed form, is suitable for use in the second stage of optimization. Based on this system, decisions are made on the use of the next blade profile in the optimization operation, correction of some zones of the median surface of the blade, complete rejection of this blade profile. In practice, it is supposed to carry out preliminary programmed measurements of coordinates, components of tangent vectors, both curvatures at a number of control points of the median surface of the blade.

\section{References}

1. P. Lampart, S. Yershov, 3D optimization of turbomachinery blading, TASK Quarterly, 6 (1) (2002)

2. R. Eisinger, A. Ruprecht, Automatic shape optimization of hydro turbine components based on CFD, TASK Quarterly, 6 (1) (2002).

3. R.A. Van den Braembussche, Turbomachinery component design by means CFD, TASK Quarterly, 6, (1) (2002).

4. I.F. Lobareva, V.A. Skorospelov, et al. On one approach to optimizing the shape of a hydraulic turbine bladem, Computational technologies, 10, (6) (2005).

5. I.F. Lobareva, S.G. Cherny, D.V. Chirkov et al. Multi-criterial optimization of the hydraulic turbine blade shape, Computational technologies, 11 (5) (2006).

6. A. Alnaga, et. al. Optimal Design of Hydraulic Turbine Distributor, WSEAS Transactions on Fluid Mechanics, 2008, 3, (2). Georgopoulou H.A., Kyriacou S.A., Giannakoglou K.C, Grafenberger P., Parkinson E. Constrained Multi-Objective Design Optimization of Hydraulic Components Using a Hierarchical Metamodel Assisted Evolutionary Algorithm. Part 1: Theory. - Proceedings of IAHR 24th Symposium on Hydraulic Machinery and Systems (Brazil, Foz do Iguassu, October 27 - 31), (2008).

7. P. Grafenberger, E. Parkinson, H.A. Georgopoulou, S.A. Kyriacou, K.C. Giannakoglou, Constrained Multi-Objective Design Optimization of Hydraulic Components Using a Hierarchical Metamodel Assisted Evolutionary Algorithm. Part 2: Applications. - Proceedings of 24th IAHR Symposium (Brazil, Foz do Iguassu, October 27 31 (2008).

8. D.V. Bannikov, S.G. Cherny, D. V. Chirkov et. al.Multimode optimization of the turnine impeller shape, Computational technologies, 14, (2) (2009).

9. J.H. Kim, H.G. Choi, Performance enhancement of axial fan blade through multi-objective optimization techniques, Journal of Mechanical Science and Technology, 24 (10) (2010).
10. L. Wang, The Optimal Design based on CFD combined with CAD for Turbine Runner. Journal of Software, 7 ( 8) (2012).

11. A.V. Semenova, D.V. Chirkov, D.A. Skorospelov, Application of the method of multi-criterial optimization for designing the shape of the impeller blade of a Kaplan hydraulic turbine, Bulletin of the Samara Scientific Center of the Russian Academy of Sciences, 15 (4) (2013).

12. F. Ayancik, U. Aradag, et. al. Hydroturbine Runner Design and Manufacturing. International Journal of Materials, Mechanics and Manufacturing, 1, (2) (2013).

13. Y. Kawajiri, Y. Enomoto, S. Kurosawa, Design optimization method for Francis turbine. Proceedings of 27th IAHR Symposium on Hydraulic Machinery and Systems IAHR 2014 ( Montreal, Canada, 22 - 26 Sept.), (2014).

14. D. Himmelblau, Applied nonlinear programming. Moskva. Mir Publisher (1975).

15. S. Finikov, Differential geometry course. Moskva. KomKniga Publisher (2006).

16. D. Rogers, J. Adams, Mathematical foundations of computer graphics. Moskva. Mir Publisher (2001). 